\title{
Improving antibiotics targeting using PCR point-of-care testing for group A streptococci in patients with uncomplicated acute sore throat
}

Ronny K Gunnarsson, Ulrich Orda, Bradley Elliott, Clare Heal, Hilary Gorges, Paul Glasziou, Chris Del Mar

\section{Background and objective}

Evidence supports some beneficial effects of antibiotics prescribed to patients with a sore throat and proven presence of group A streptococci (GAS).

\section{Methods}

A total of 283 patients were included from North and North-West Queensland, Australia, at their first presentation for uncomplicated acute sore throat. Patterns of antibiotic prescribing were explored before and after testing for GAS using a rapid point-of-care polymerase chain reaction (PCR) test.

\section{Results}

The results of the study showed the Australian Therapeutic Guidelines were often not adhered to. The PCR test reduced the proportion of patients prescribed antibiotics from $46 \%$ to $40 \%$. The decision to prescribe antibiotics was changed in $30 \%$ of patients $(P<0.001)$ : before testing only $40 \%$ of patients prescribed antibiotics had a positive GAS PCR while this increased to $97 \%$ after testing.

\section{Discussion}

An easy-to-use point-of-care test to detect GAS allows better targeting of antibiotic prescribing in patients with an uncomplicated acute sore throat.
ACUTE SORE THROAT is a common reason for presenting to primary healthcare. ${ }^{1}$ Although most cases of a sore throat are viral in origin, a sore throat caused by group A $\beta$-haemolytic streptococci (GAS) can occasionally have suppurative complications as well as significant non-suppurative sequelae such as rheumatic fever and glomerulonephritis. The risk for rheumatic fever is very low in most high-income countries but may be higher in low-income countries. ${ }^{2}$ Australia and New Zealand are high-income countries that incur a high incidence of rheumatic fever, especially among people living remotely and Aboriginal and Torres Strait Islander peoples in Australia and Maori peoples in New Zealand. ${ }^{3,4}$

\section{Aetiology of the sore throat}

The aetiology of the majority of sore throats is a viral infection, but a clinically relevant number of cases are caused by GAS. Other bacterial aetiologies potentially involved are Streptococcus dysgalactiae subspecies Equisimilis (SDSE), ${ }^{5-7}$ Fusobacterium necrophorum ${ }^{8,9}$ or other bacteria. ${ }^{10}$ SDSE was previously labelled group C $\beta$-haemolytic streptococci or group $\mathrm{G} \beta$-haemolytic streptococci. However, systematic reviews focusing on case-control studies have not established that SDSE is linked to the uncomplicated acute sore throat to the extent that it should be considered in the management of these patients. ${ }^{11-14}$

\section{Antibiotic treatment of patients} with an acute sore throat Antibiotics reduce acute symptoms of sore throats in children, ${ }^{15-17}$ and in children and adults combined, if GAS is present. ${ }^{18-20}$ Although antibiotic treatment seems effective in primary prevention of rheumatic fever, ${ }^{3,21,22}$ the magnitude of the effect on acute symptoms of an uncomplicated acute sore throat is modest. ${ }^{23}$ Most current studies show no effect in children ${ }^{15,16}$ and children and adults combined ${ }^{18}$ if GAS is not present. The exceptions are one study by Petersen, ${ }^{24}$ focusing on GAS-negative patients, and a study by Zwart, ${ }^{25}$ focusing on patients with presence of SDSE and absence of GAS. Both showed a borderline effect on symptoms of an acute sore throat in adults with $P$ values of 0.049 and 0.05 , respectively. There are, to date, no studies of antibiotic treatment for an uncomplicated sore throat suspected to be caused by F. necrophorum.

All studies that compared the effect of antibiotics with placebo, and performed a separate analysis for GAS-positive and GAS-negative patients, found an effect in GAS-positive patients and none in those who were GAS-negative. ${ }^{15,16,18,20}$ These studies show there is firm evidence in children and adults for a modest effect of antibiotics on the acute symptoms 
of acute sore throat if GAS is present. There is only very weak support for an effect in adults if GAS is not present, and no evidence for children with no proven growth of GAS. Furthermore, the reduction in risk for rheumatic fever and the modest reduction in acute symptoms proven in previous intervention studies must be weighed against potential side effects of antibiotic treatment.

\section{Symptomatic carriers and testing presence of GAS}

GAS may sometimes be present in healthy individuals, who are labelled 'asymptomatic carriers of GAS'. An incorrect conclusion about the aetiology of symptoms might be drawn if these asymptomatic carriers of GAS acquire a viral pharyngitis and become symptomatic carriers of GAS. ${ }^{26,27}$ Patients attending for a sore throat are a mixture of those ill from a virus, symptomatic carriers of GAS, those ill from GAS and those ill from other bacteria. ${ }^{26,27}$ Tests for presence of GAS cannot distinguish between symptomatic carriers of GAS and those ill from GAS.

The negative predictive value of a test to detect GAS, while also considering symptomatic carriers, is always very high (97-99\%). ${ }^{26,28,29}$ Most tests for presence of GAS will be negative in this mixture of patients and this is very effective at ruling out GAS as the cause of the sore throat, even in the presence of symptomatic carriers ill from something other than GAS. ${ }^{26,28,29}$ Hence, a negative point-ofcare test (POCT) can be used to stop an intended antibiotic prescribing in uncomplicated cases of acute sore throat. In most scenarios, this is the main value of a POCT to detect GAS. ${ }^{29}$

The clinical value of a positive test for GAS varies depending on the proportion of symptomatic carriers of GAS. ${ }^{26,28}$ In most scenarios, a positive test for GAS in a patient with a sore throat has a high probability of indicating the aetiology. ${ }^{28}$

\section{Clinical evaluation and point-of-care testing Many different clinical scoring algorithms can be used to try to identify patients}

for whom antibiotic treatment may be beneficial. The most widely used is the Centor criteria. ${ }^{30}$ All clinical scoring algorithms, including the Centor criteria, have inherent low sensitivity and specificity. ${ }^{31}$ Relying solely on the Centor criteria means many patients ill from GAS are left without antibiotics while many patients ill from a virus are prescribed antibiotics. $^{32}$

Given the current evidence for the effect of antibiotic treatment in patients with an uncomplicated acute sore throat, it seems reasonable to emphasise identifying the presence of GAS before an antibiotic prescription. ${ }^{29} \mathrm{~A}$ throat swab sent for culture is of limited value for the decision to prescribe antibiotics because of the long delay before the result is available. ${ }^{33}$ However, analysing the throat swab with a high-quality rapid POCT, delivering a result while the patient is still at the premises, might be very useful. ${ }^{28,29,32}$ Modern POCT for GAS have a sensitivity and specificity to detect GAS on par with, or even better than, conventional culture techniques. ${ }^{34}$

The objective of the present study was to investigate to what extent the introduction of a high-sensitive polymerase chain reaction (PCR) POCT to detect presence of GAS changes the management of otherwise healthy patients attending for an uncomplicated acute sore throat.

\section{Methods}

This prospective study was approved 5 March 2018 by the Townsville Hospital and Health Service Human Research Ethics Committee (registration number HREC/17/QTHS/246).

\section{Study design}

Patients at three sites in North and North-West Queensland, Australia, attending for a sore throat, and their treating medical practitioner, were asked to participate in a prospective observational study.

\section{Study objectives}

The study aimed to clarify to what extent medical practitioners' decisions to prescribe antibiotics are changed by a POCT. The study also aimed to explore factors associated with prescribing antibiotics despite knowing a PCR test indicates no presence of GAS.

\section{Practitioners, patients and recruitment} All medical practitioners at the three sites managing patients with an acute uncomplicated sore throat were invited to participate. Practitioners were asked to consider including all consecutive patients attending Mt Isa Base Hospital emergency department, Hinchinbrook Health Care in Ingham or One Stop Medical in Mackay who presented with a main complaint of an acute sore throat, unless it was a representation for the same illness episode or if the medical practitioner deemed the patient so ill that admission to hospital was required. Hinchinbrook Health Care and One Stop Medical are primary healthcare centres. The emergency department in Mt Isa typically manages acute emergency department patients, but also lower acuity problems, including patients presenting with a sore throat.

\section{Data collection}

Data were collected on a case report form (CRF) based on a CRF that worked well in a previous study. ${ }^{28,32}$ On the first page of the CRF, information was retrieved about the treating medical practitioner's level of education and patient's age, sex, cultural identity, symptoms and signs, and the medical practitioner's clinical diagnosis based on symptoms and signs. Retrieved information made it possible to provide a Centor score on each patient as a pre-test probability of the patient having GAS present. The Centor score consists of four clinical signs or symptoms, each adding to a total Centor score from zero to four: tonsillar exudates, swollen tender anterior cervical nodes, lack of a cough and a history of fever. ${ }^{30}$ Finally, the medical practitioner stated if they would prescribe antibiotics on the basis of the clinical assessment.

A throat swab for PCR POCT was performed after the first page of the CRF was completed and the outcome was registered at the top of page two. Swabs were taken either by the medical practitioner or a trained nurse. Medical practitioners, and in some sites nurses, 
were already taking throat swabs and had long experience of doing this. In addition, they were also informed in writing about the best technique to obtain an optimal throat swab sample. Nurses were employed by the clinic. After obtaining the test result, the CRF required the medical practitioner to note on page two if they would prescribe antibiotics knowing the test outcome. The medical practitioner was also asked about the main reason for prescribing antibiotics despite a negative test (if that was the case).

Any antibiotic prescription was classified by the medical practitioner as for immediate consumption or delayed consumption, meaning the patient was instructed to start medication later if symptoms remained or became worse. The exact recommended delay may have varied between practitioners and this was not registered.

The CRF had no code or signature linking the information to a particular patient or treating medical practitioner. Hence, the information collected on the $\mathrm{CRF}$ was anonymous.

\section{Test for presence of GAS}

Before this study, neither Hinchinbrook Health Care nor One Stop Medical had used a POCT in managing patients with a sore throat. The Mt Isa Hospital emergency department had previously used a rapid antigen detection test as a POCT for children attending with a sore throat when they participated in a study during the period June 2014 to February 2015. ${ }^{28,32}$

All patients were swabbed and the presence of GAS was analysed using Abbott ID NOW Strep A (formerly Alere i Strep). This is a nucleic acid test using LAMP technology that can be described as an isothermal PCR with similar test characteristics as conventional PCR. This test has a $>95 \%$ sensitivity and $>95 \%$ specificity to detect GAS when compared with in-house $\mathrm{PCR}^{35}$ or culture. ${ }^{36}$ Participating medical practitioners were informed about the characteristics of this test. Nurses at each clinic received a one-hour training session from Abbott (formerly Alere) to run the test. Nurses were employed by the clinic. The test took approximately eight minutes to analyse, after which time the patient returned to the medical practitioner. This test was at the time of the study approved by the Australian Therapeutic Goods Administration for use in patients with a sore throat.

\section{Statistical analysis}

Antibiotic prescribing patterns before and after the outcome of the PCR test for GAS were presented with descriptive statistics. Sites were compared using two-sided chi-square or t-test (Appendices, available online). Data for antibiotics for immediate or delayed consumption were merged and chi-square was used to test the hypothesis that the POCT resulted in a changed prescribing behaviour. A multivariable logistic regression was used to clarify if the medical practitioner's level of training, patient's age, patient's sex, patient's cultural identity or the Centor scores were associated with an increased risk of prescribing antibiotics despite proven absence of GAS defined as a negative test outcome of the PCR test.

\section{Sample size calculation}

A sample size calculation was made for the potential difference in antibiotic prescribing in case of a negative test for GAS. It was assumed that $20 \%$ of general practice trainees (registrars) would prescribe antibiotics despite a negative test for GAS, in comparison to $40 \%$ for specialist general practitioners, assuming a level of significance of 0.05 , a power of 0.8 and a two-sided test requires 207 patients.

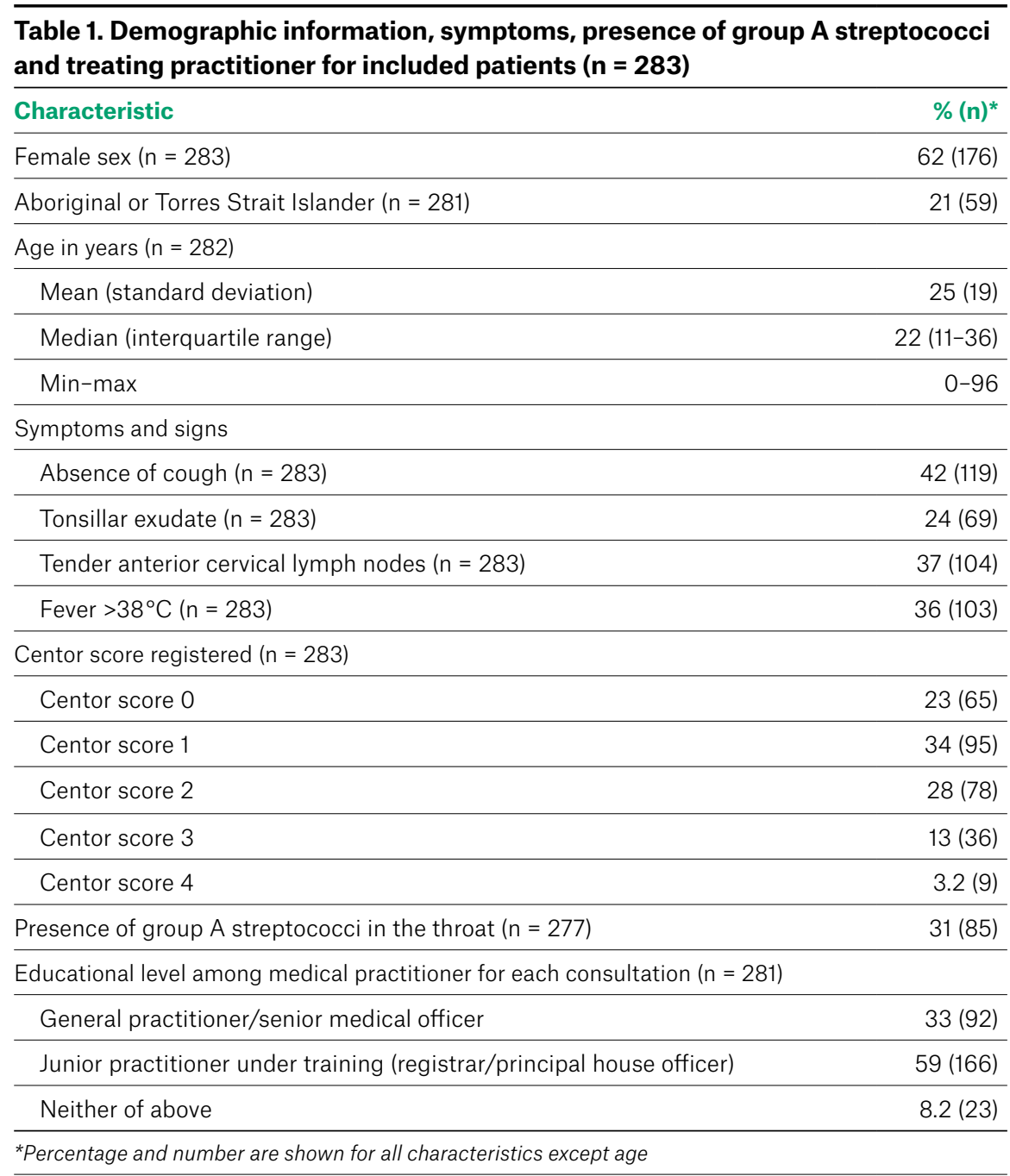


The software $G^{*}$ Power version 3.1.9.2 was used assuming logistic regression with antibiotic prescribing as the dependent variable. ${ }^{37,38}$ The researchers aimed to collect data from 300 patients.

\section{Role of the funding source}

This study was funded by James Cook University and Bond University, both in Australia. Some of the authors are employed by these universities. The PCR devices and test kits were purchased from Abbott. Hence, Abbott was not a funder and had no influence on study design, analysis of data or writing of the manuscript.

\section{Patient and public involvement}

Neither patients nor the public were involved in the design of this study.

\section{Results}

Two hundred and eighty-three patients were included from April 2018 to February 2019. Recruitment ceased as the available test kits expired. Half of the patients were between 11 and 36 years with a slight predominance of females (Table 1; Appendix 1, available online only). The Mount Isa site had, as expected, a larger proportion of Aboriginal and Torres Strait Islander patients (Appendix 1). The PCR test showed presence of GAS in 37\% (27/57) of Aboriginal and Torres Strait Islander patients, while it was only positive in $25 \%$ (50/204) of Caucasian patients $(P=0.00082$, chi-square $)$. There was no sex difference in the proportion of positive tests for presence of GAS $(P=0.35$, chi-square) .

\section{Pre-test prescribing intention}

Before the PCR POCT, almost all patients perceived to have a bacterial infection were prescribed antibiotics (Figure 1). This included patients with 0-2 Centor scores, of whom $37 \%$ were prescribed antibiotics (Figure 1). Antibiotics were prescribed to $52 \%(31 / 59)$ of Aboriginal and Torres Strait Islander patients and to $44 \%(92 / 208)$ of Caucasian patients $(P=0.26$, chi-square).

\section{Change incurred by introducing the PCR test}

The outcome of the PCR test as well as the pre-test and post-test antibiotic prescribing was recorded for 276 patients (Table 2; Appendix 2, available online only). Patients were prescribed antibiotics both pre- and post-test in $81 / 276(29 \%)$ of cases while $112 / 276$

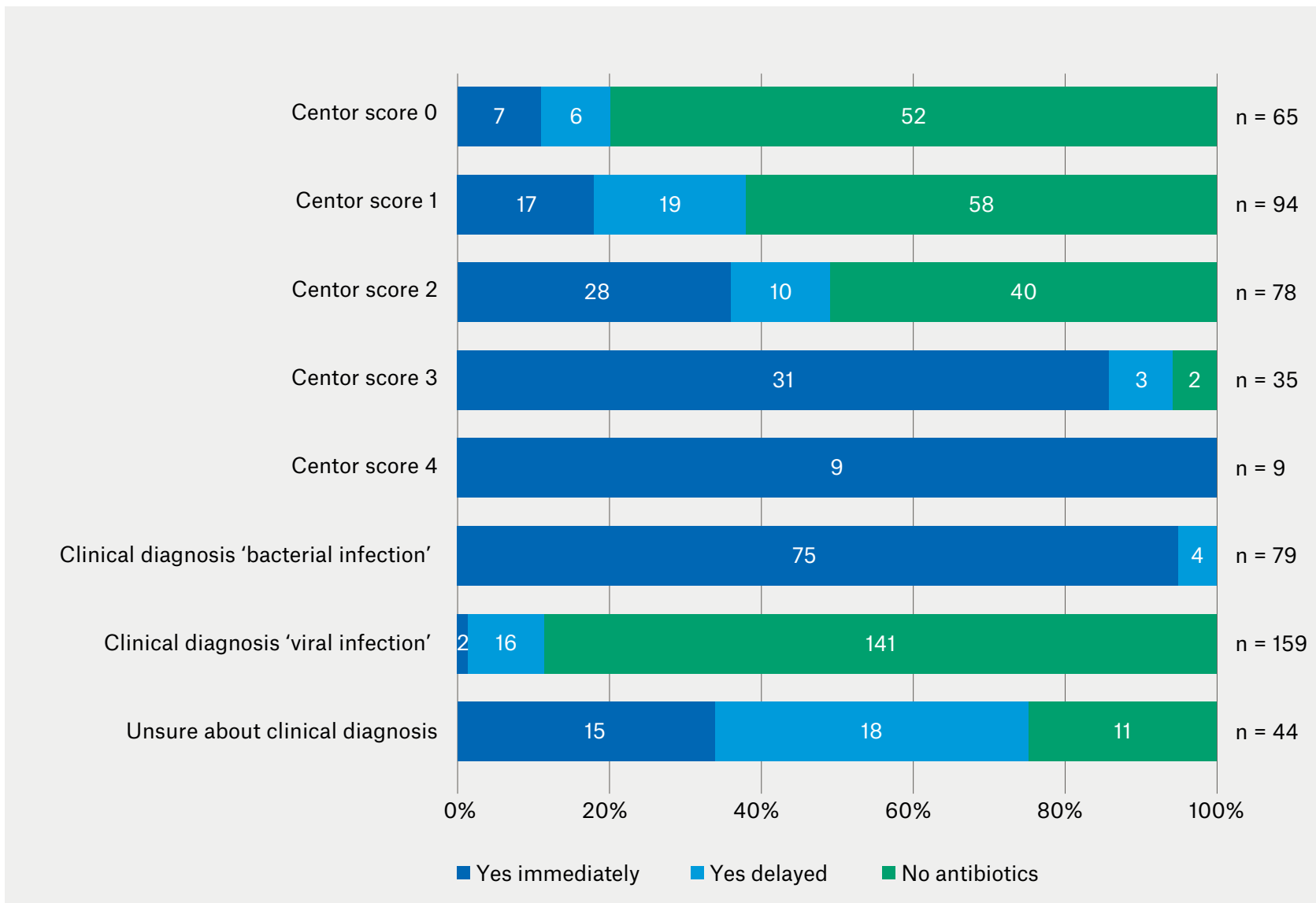

Figure 1. Antibiotic prescribing pattern before result of polymerase chain reaction point-of-case test for group A streptococci was available 
(41\%) were not prescribed antibiotics pre- or post-test. However, the decision regarding antibiotic prescribing was changed for $83 / 276(30 \%)$ of patients $(P<0.001$, chi-square; Table 2$)$. Hence, introducing the PCR POCT only reduced the proportion of patients prescribed antibiotics from $46 \%$ to $40 \%$; but the management was actually changed for $30 \%$ of patients. The proportion of patients prescribed antibiotics that most likely had GAS in their throats increased from $51 / 128$ (40\%) to $114 / 117$ (97\%)

(Table 2). Furthermore, those patients with a sore throat with GAS in their throat and not being prescribed antibiotics dropped from $40 \%(34 / 85)$ pre-test to $1.2 \%(1 / 85)$ post-test.

\section{Antibiotic prescribing in case of a negative test for presence of GAS}

Testing for GAS was negative in 69\% (192/277) of patients. In 77/192 patients this was unexpected and the practitioner pre-test had intended to prescribe antibiotics either immediately $(n=49)$ or delayed $(n=28)$. The treating medical practitioners still decided to prescribe antibiotics for 30 of these 77 patients, despite a negative PCR POCT. The reasons stated for this were: that the medical practitioner suspected the patient had a throat infection caused by a bacteria other than GAS $(n=17)$, that the patient was ill from GAS despite a negative PCR test $(n=1)$, request from the patient $(n=1)$, throat swab was difficult to obtain $(n=1)$, patient was diagnosed with serous otitis media/glue ear $(n=1)$, patient was diagnosed with 'chest infection' $(n=1)$ and no reason was stated for eight patients.

Multivariable logistic regression $(n=190)$ revealed that an increase in Centor score by one increased the chance of antibiotic prescribing, despite a negative PCR test for presence of GAS, with an odds ratio of 2.2 (95\% confidence interval [CI]: $1.4,3,5 ; P=0.00091$; Table 3). Furthermore, specialist general practitioners were more prone to prescribe antibiotics despite a negative PCR test for GAS, compared with general practice trainees (registrars), with an odds ratio of 4.8 (95\% CI: $1.8,12 ; P=0.0013$;
Table 3). No other variables were associated with antibiotic prescribing despite a negative test for GAS.

\section{Antibiotic prescribing in case of an unexpected positive test for presence of GAS}

The test for GAS was unexpectedly positive among 34 patients where the practitioner pre-test had chosen to not prescribe antibiotics. This resulted in an antibiotic prescription in 33 of these 34 patients (Table 2 ).

\section{Discussion}

This study found that introducing a PCR POCT test helped practitioners direct antibiotic prescribing to patients with a sore throat and GAS in their throats. This meant that many patients not prescribed antibiotics pre-test were prescribed antibiotics post-test and vice versa. Overall, the management was changed for $30 \%$ of patients, with a shift from prescribing antibiotics to patients without GAS to those with GAS. The overall reduction in patients prescribed

\section{Table 2. Change of antibiotic prescribing pattern after result of test for group $A$ streptococci was available}

\begin{tabular}{llrrrr}
\hline Pre-test antibiotic prescribing & PCR test for GAS & \multicolumn{3}{l}{ Post-test antibiotic prescribing* } \\
\hline No & & No & YD & YI & $\mathbf{n}$ \\
\hline & Negative & 111 & $\mathbf{2}$ & $\mathbf{1}$ & 114 \\
\cline { 2 - 6 } & Positive & 1 & 0 & $\mathbf{3 3}$ & 34 \\
\hline YD & Negative & $\mathbf{2 3}$ & 4 & $\mathbf{1}$ & 28 \\
\cline { 2 - 6 } & Positive & 0 & 1 & $\mathbf{9}$ & 10 \\
\hline YI & Negative & $\mathbf{2 4}$ & $\mathbf{2}$ & 23 & 49 \\
\cline { 2 - 6 } & Positive & 0 & 0 & 41 & 41 \\
\hline
\end{tabular}

${ }^{*}$ Changes from pre- to post-test are marked bold

GAS, group A streptococci; No, no antibiotics; $P C R$, polymerase chain reaction; YD, antibiotics prescribed delayed; $Y I$, antibiotics prescribed for immediate consumption

Table 3. Factors associated with antibiotic prescribing in patients with a sore throat and no presence of group A streptococci $(n=190)$

Adjusted odds ratio

(95\% confidence interval) $\quad P$ value

Level of training of medical practitioner

General practitioner (GP)/senior medical officer (SMO)

\begin{tabular}{lrr}
\hline Neither GP/SMO nor registrar & $1.2(0.12,12)$ & 0.87 \\
\hline Registrar under education & (Reference) & 0.0051 \\
\hline Increase of patient's age (one year) & $0.99(0.97,1.0)$ & 0.55 \\
\hline Female sex of the patient & $0.85(0.35,2.0)$ & 0.71 \\
\hline
\end{tabular}

Cultural identity

\begin{tabular}{lrr}
\hline Aboriginal or Torres Strait Islander & $2.2(0.17,29)$ & 0.54 \\
\hline Caucasian & $1.3(0.13,13)$ & 0.83 \\
\hline Other & (Reference) & 0.69 \\
\hline Increase of Centor score one step & $2.2(1.4,3.5)$ & 0.00091 \\
\hline
\end{tabular}


antibiotics was modest, but antibiotics were better targeted to patients likely to be ill from GAS. Hence, the overall value of POCT testing would be from the improved targeting of antibiotic prescriptions, not the overall reduction.

A substantial proportion of patients with 0-2 Centor criteria were prescribed antibiotics both pre- and post-testing for presence of GAS. A negative PCR often resulted in an antibiotic prescription and an unexpected positive PCR test almost always resulted in an antibiotic prescription. Senior medical practitioners were much more likely to prescribe antibiotics to patients with no presence of GAS than junior practitioners, perhaps because junior practitioners have encountered POCT during their recent training, making them trust the POCT more, or they may have a more recent education in antibiotic stewardship. All these findings should trigger a healthy discussion about the need for a changed behaviour among medical practitioners.

\section{Strength and limitations}

This study did not only investigate the total reduction in antibiotic prescribing, which was modest, but also the change towards more targeted antibiotic prescribing, which was large. This is highly relevant for patients at high risk for developing rheumatic fever. The rate of positive tests for GAS was higher in this study (31\%), than in many other studies of patients with a sore throat in primary care. The total reduction in antibiotic prescribing would probably be more pronounced in settings with a lower prevalence of GAS.

A potential limitation is that recruitment was stopped before the target of 300 patients was achieved. However, the study managed to recruit well over the 207 patients estimated by the sample size calculation and it was deemed unnecessary to apply for further funding to purchase more test kits.

Information retrieved on the $\mathrm{CRF}$ was anonymous, and one consequence is that the exact number of medical practitioners participating in the study in unknown. Another potential limitation is that medical practitioners may have ignored the study in times of high patient load and not all consecutive suitable patients are likely to have been invited. However, the allocation between Centor scores in Table 1 is similar to what is seen in other recent studies with few patients fulfilling all four Centor criteria, ${ }^{32,39}$ suggesting that this problem is unlikely to have introduced a systematic error.

Finally, the less widely used but more recent McIsaac scores were not used in this study. The difference between the Centor and McIsaac scores to select patients at high probability for a GAS sore throat is marginal. ${ }^{40}$ The results reflect short-term intended behaviours, and it is not known how this may change with continued usage.

\section{Presence of GAS and POCT}

Guidelines suggesting antibiotic treatment on clinical grounds irrespective of presence of GAS or $\mathrm{SDSE}^{7}$ makes POCT irrelevant. The consequence is an encouragement to rely on clinical symptoms and signs rather than the more objective throat swab. This behaviour significantly increases antibiotic prescribing ${ }^{29,32}$ and leaves a significant proportion of patients ill from GAS, even in areas with a high incidence of rheumatic fever, without antibiotics, ${ }^{32}$ which is not acceptable. The latter is emphasised by the fact that testing reduced the proportion of patients with GAS not prescribed antibiotics from $40 \%$ to $1.2 \%$.

\section{Availability of POCT}

The cost of POCT includes costs for maintaining approval with regulatory authorities and balancing stocking an adequate number of test kits with wastage due to expiry dates. Countries such as Australia, where POCT is not recommended in primary care guidelines, are likely to be considered a dead market and the registration may be withdrawn. Hence, the recommendations suggested above are unlikely to be successful in Australia unless the therapeutic guideline for management of patients with a sore throat $^{41}$ is updated to include POCT.

The requirement for accreditation with a continuous quality assurance process is a limiting factor. This would be simple to set up if an organisation would take responsibility for arranging dummy samples that could be sent to clinics for analysis. This is currently unavailable in Australia. Another limiting factor is that the overall cost for equipment, test kits, accreditation and staff time does not match the current reimbursement from the Medicare Benefits Schedule (MBS). It is likely that POCTs providing early accurate diagnosis and subsequent treatment would result in better outcomes, fewer hospital admissions and fewer lost days of productivity and this would be an argument for introducing an adequate MBS reimbursement for such tests. Although, this should be confirmed in a health economic analysis.

\section{Conclusions and implications for general practice}

Introducing a POCT to detect GAS made antibiotic prescribing much more targeted from prescribing to patients with no GAS present to those having GAS. Consequently, the POCT significantly reduced the risk for patients with GAS being left without antibiotics; this is especially important when the risk for rheumatic fever must be considered. The most-used guideline in primary healthcare in Australia ${ }^{41}$ does not mention POCTs, so most practitioners in Australia are unfamiliar with them. As Tarca et $\mathrm{l}^{42}$ previously suggested, there is also a need for an evidence-based single Australian national sore throat guideline where the role of POCTs to detect GAS is clarified.

\footnotetext{
Authors

Ronny K Gunnarsson MD, PhD, Adjunct Professor, General Practice/Family Medicine, School of Public Health and Community Medicine, Institute of Medicine, Sahlgrenska Academy, University of Gothenburg, Gothenburg, Sweden; General Practice/Family, Region Västra Götaland, Research and Development Primary Health Care, Research and Development Center Södra Älvsborg, Sweden; General Practice/Family, Centre for Antibiotic Resistance Research (CARe) at University of Gothenburg, Gothenburg, Sweden

Ulrich Orda MD, PhD, Director of Emergency Medicine Department, Mount Isa Hospital/North West Hospital and Health Service, Qld; Associate Professor, College of Medicine and Dentistry, James Cook University, Qld

Bradley Elliott FRACGP, BBSc, General Practice/ Family Medicine, Hinchinbrook Health Care, Qld Clare Heal MBChB, MPHTM, PhD, Professor in General Practice, College of Medicine and Dentistry, James Cook University, Qld
} 
Hilary Gorges MD, FRACGP, Senior Lecturer, College of Medicine and Dentistry, James Cook University, Qld; General Practitioner; South Side Medical, Qld Paul Glasziou MBBS, PhD, Professor in General Practice, Centre for Research in Evidence-Based Practice, Bond University, Qld

Chris Del Mar MBBChir, MA, MD, FRAGCP, Professor in General Practice, Centre for Research in EvidenceBased Practice, Bond University, Qld

Competing interests: $P G$ reports that he is a co-investigator on a National Health and Medical Research Council (NHMRC) grant examining ways to improve antibiotic prescribing in primary care. CDM reports grants for research into antibiotic resistance and antibiotic use in primary care from the NHMRC, outside the submitted work.

Funding: None.

Provenance and peer review: Not commissioned, externally peer reviewed.

\section{Correspondence to:}

ronny.gunnarsson@infovoice.se

\section{References}

1. Cherry DK, Woodwell DA. National ambulatory medical care survey: 2000 summary. Adv Data 2002;(328):1-32.

2. Seckeler MD, Hoke TR. The worldwide epidemiology of acute rheumatic fever and rheumatic heart disease. Clin Epidemiol 2011;3:67-84. doi: 10.2147/CLEP.S12977.

3. Lennon D, Anderson P, Kerdemilidis M, et al. First presentation acute rheumatic fever is preventable in a community setting: $A$ school-based intervention. Pediatr Infect Dis J 2017;36(12):1113-18. doi: 10.1097/ INF.0000000000001581.

4. Australian Institute of Health and Welfare. Rheumatic heart disease and acute rheumatic fever in Australia: 1996-2012. Cardiovascular disease series. Cat. no. CVD 60. Canberra, ACT: AlHW, 2013.

5. Lindbaek M, Høiby EA, Lermark G, Steinsholt IM, Hjortdahl P. Clinical symptoms and signs in sore throat patients with large colony variant betahaemolytic streptococci groups $\mathrm{C}$ or $\mathrm{G}$ versus group A. Br J Gen Pract 2005;55(517):615-19.

6. Tiemstra J, Miranda RL. Role of non-group A streptococci in acute pharyngitis. J Am Board Fam Med 2009;22(6):663-69. doi: 10.3122/ jabfm.2009.06.090035.

7. Little P, Moore M, Hobbs FD, et al. PRImary care Streptococcal Management (PRISM) study: Identifying clinical variables associated with Lancefield group A $\beta$-haemolytic streptococci and Lancefield non-Group A streptococcal throat infections from two cohorts of patients presenting with an acute sore throat. BMJ open 2013;3(10):e003943. doi: 10.1136/bmjopen-2013003943.

8. Lemierre A. On certain septicaemias due to anaerobic organisms. Lancet 1936;227(5874):701-03. doi: 10.1016/S0140-6736(00)57035-4.

9. Wright WF, Shiner CN, Ribes JA. Lemierre syndrome. South Med J 2012;105(5):283-88. doi: 10.1097/SMJ.0b013e31825581ef.

10. Gunnarsson RK, Holm SE, Söderström M. The prevalence of potential pathogenic bacteria in nasopharyngeal samples from individuals with a respiratory tract infection and a sore throat - Implications for the diagnosis of pharyngotonsillitis. Fam Pract 2001;18(3):266-71. doi: 10.1093/fampra/18.3.266
11. Cimolai N, Elford RW, Bryan L, Anand C, Berger P. Do the beta-hemolytic non-group $A$ streptococci cause pharyngitis? Rev Infect Dis 1988;10(3):587-601. doi: 10.1093/clinids/10.3.587.

12. Arditi M, Shulman ST, Davis AT, Yogev R. Group $\mathrm{C}$ beta-hemolytic streptococcal infections in children: Nine pediatric cases and review. Rev Infect Dis 1989;11(1):34-45. doi: 10.1093/ clinids/11.1.34.

13. Marchello $\mathrm{C}$, Ebell MH. Prevalence of group $\mathrm{C}$ streptococcus and Fusobacterium necrophorum in patients with sore throat: A meta-analysis. Ann Fam Med 2016;14(6):567-74. doi: 10.1370/ afm.2005.

14. Gunnarsson RK, Manchal N. Group C beta haemolytic streptococci as a potential pathogen in patients presenting with an uncomplicated acute sore throat - A systematic literature review and meta-analysis. Scand J Prim Health Care 2020;38(2):226-37. doi: 10.1080/02813432.2020.1753374.

15. Pichichero ME, Disney FA, Talpey WB, et al. Adverse and beneficial effects of immediate treatment of Group A beta-hemolytic streptococcal pharyngitis with penicillin. Pediatr Infect Dis J 1987;6(7):635-43. doi: 10.1097/00006454-198707000-00004.

16. Krober MS, Bass JW, Michels GN. Streptococcal pharyngitis. Placebo-controlled double-blind evaluation of clinical response to penicillin therapy. JAMA 1985;253(9):1271-74. doi: 10.1001/ jama.253.9.1271.

17. Nelson JD. The effect of penicillin therapy on the symptoms and signs of streptococcal pharyngitis. Pediatr Infect Dis 1984;3(1):10-13. doi: 10.1097/00006454-198401000-00004.

18. De Meyere M, Mervielde Y, Verschraegen G, Bogaert M. Effect of penicillin on the clinical course of streptococcal pharyngitis in general practice. Eur J Clin Pharmacol 1992;43(6):581-85. doi: 10.1007/BF02284954.

19. Middleton DB, D'Amico F, Merenstein JH. Standardized symptomatic treatment versus penicillin as initial therapy for streptococcal pharyngitis. J Pediatr 1988;113(6):1089-94. doi: 10.1016/s0022-3476(88)80588-2.

20. Dagnelie CF, van der Graaf Y, De Melker RA. Do patients with sore throat benefit from penicillin? A randomized double-blind placebo-controlled clinical trial with penicillin $\mathrm{V}$ in general practice. Br J Gen Pract 1996;46(411):589-93.

21. Catanzaro FJ, Stetson CA, Morris AJ, et al. The role of the streptococcus in the pathogenesis of rheumatic fever. Am J Med 1954;17(6):749-56. doi: 10.1016/0002-9343(54)90219-3.

22. Robertson KA, Volmink JA, Mayosi BM. Antibiotics for the primary prevention of acute rheumatic fever: A meta-analysis. BMC Cardiovasc Disord 2005;5(1):11. doi: 10.1186/14712261-5-11.

23. Spinks A, Glasziou PP, Del Mar CB. Antibiotics for sore throat. Cochrane Database Syst Rev 2013;(11):CD000023. doi: 10.1002/14651858. CD000023.pub4

24. Petersen K, Phillips RS, Soukup J, Komaroff AL, Aronson M. The effect of erythromycin on resolution of symptoms among adults with pharyngitis not caused by group A streptococcus. J Gen Intern Med 1997;12(2):95-101. doi: 10.1046/j.1525-1497.1997.00013.x.

25. Zwart S, Sachs AP, Ruijs GJ, Gubbels JW, Hoes AW, de Melker RA. Penicillin for acute sore throat: Randomised double blind trial of seven days versus three days treatment or placebo in adults. BMJ 2000;320(7228):150-54. doi: 10.1136/ bmj.320.7228.150.

26. Gunnarsson RK, Lanke J. The predictive value of microbiologic diagnostic tests if asymptomatic carriers are present. Stat Med 2002;21(12):1773-85. doi: 10.1002/sim.1119.

27. Gunnarsson RK. Microbiologic diagnostic tests when asymptomatic carriers are present: Aspects of the use of conventional throat and nasopharyngeal cultures as examples. Gothenburg, SE: Göteborg University, 2001.

28. Orda U, Gunnarsson RK, Orda S, Fitzgerald M, Rofe G, Dargan A. Etiologic predictive value of a rapid immunoassay for detection of Group A streptococci antigen from throat swabs on patients presenting with a sore throat. Int J Infect Dis 2016;45:32-35. doi: 10.1016/j.ijid.2016.02.002.

29. Gunnarsson MS, Sundvall PD, Gunnarsson R. In primary health care, never prescribe antibiotics to patients suspected of having an uncomplicated sore throat caused by group A beta-haemolytic streptococci without first confirming the presence of this bacterium. Scand J Infect Dis 2012;44(12):915-21. doi: 10.3109/00365548.2012.700768.

30. Centor RM, Witherspoon JM, Dalton HP, Brody CE, Link K. The diagnosis of strep throat in adults in the emergency room. Med Decis Making 1981;1(3):239-46. doi: 10.1177/0272989X8100100304

31. Aalbers J, O'Brien KK, Chan WS, et al. Predicting streptococcal pharyngitis in adults in primary care: A systematic review of the diagnostic accuracy of symptoms and signs and validation of the Centor score. BMC Med 2011;9:67. doi: 10.1186/1741-7015-9-67.

32. Orda U, Mitra B, Orda S, et al. Point of care testing for group A streptococci in patients presenting with pharyngitis will improve appropriate antibiotic prescription. Emerg Med Australas 2016;28(2):199-204. doi: 10.1111/1742-6723.12567.

33. Cheung L, Pattni V, Peacock P, Sood S Gupta D. Throat swabs have no influence on the management of patients with sore throats. J Laryngol Otol 2017;131(11):977-81. doi: 10.1017/ S002221511700189X.

34. Lindbaek M, Høiby EA, Lermark G, Steinsholt IM, Hjortdahl P. Which is the best method to trace group A streptococci in sore throat patients: Culture or GAS antigen test? Scand J Prim Health Care 2004;22(4):233-38. doi: 10.1080/02813430410006675.

35. Cohen DM, Russo ME, Jaggi P, Kline J, Gluckman W, Parekh A. Multicenter clinical evaluation of the novel Alere i Strep A isothermal nucleic acid amplification Test. J Clin Microbiol 2015;53(7):2258-61. doi: 10.1128/JCM.00490-15.

36. Weinzierl EP, Jerris RC, Gonzalez MD, Piccini JA, Rogers BB. Comparison of Alere i Strep A rapid molecular assay with rapid antigen testing and culture in a pediatric outpatient setting. Am J Clin Pathol 2018;150(3):235-39. doi: 10.1093/ajcp/ aqy038.

37. Faul F, Erdfelder E, Lang AG, Buchner A. $G^{*}$ Power 3: A flexible statistical power analysis program for the social, behavioral, and biomedica sciences. Behav Res Methods 2007;39(2):175-91. doi: 10.3758/bf03193146.

38. Faul F, Erdfelder E, Buchner A, Lang AG. Statistical power analyses using $G^{*}$ Power 3.1: Tests for correlation and regression analyses. Behav Res Methods 2009;41(4):1149-60. doi: 10.3758/BRM.41.4.1149. 
39. Centor RM, Atkinson TP, Ratliff AE, et al. The clinical presentation of Fusobacterium-positive and streptococcal-positive pharyngitis in a

university health clinic: A cross-sectional study.

Ann Intern Med 2015;162(4):241-47. doi: 10.7326/

M14-1305.

40. Fine AM, Nizet V, MandI KD. Large-scale validation of the Centor and Mclsaac scores to predict group A streptococcal pharyngitis. Arch Intern Med 2012;172(11):847-52. doi: 10.1001/ archinternmed.2012.950.

41. Expert Group for Antibiotic. Acute pharyngitis and/or tonsillitis. In: eTG complete [Internet]. West Melbourne, Vic: Therapeutic Guidelines Limited, 2014.

42. Tarca AJ, Hand RM, Wyber R. Call for national sore throat guideline. Med J Aust 2019;210(10):477-e1. doi: 10.5694/mja2.50166. 